\title{
Patrones de distribución de la oferta turística mediante técnicas geoestadísticas en Extremadura (2004-2014)
}

Distribution patterns of tourism supply in Extremadura using geostatistical techniques (2004-2014)

\author{
José Manuel Sánchez Martín \\ jmsanche@unex.es \\ Departamento de Arte y Ciencias del Territorio \\ Universidad de Extremadura (España) \\ Marcelino Sánchez Rivero \\ sanriver@unex.es \\ Departamento de Economía \\ Universidad de Extremadura (España) \\ Juan Ignacio Rengifo Gallego \\ irengifo@unex.es \\ Departamento de Arte y Ciencias del Territorio \\ Universidad de Extremadura (España)
}

\section{Resumen}

El rápido incremento de la oferta de alojamientos en Extremadura no se ha visto correspondido con un aumento armónico en el número de visitantes y de pernoctaciones, hecho que ha provocado un desajuste notable entre la oferta y la demanda. El aumento de oferta se ha producido de forma desigual sobre el territorio, por lo que esta investigación pretende determinar los patrones de 
distribución que se detectan en los tres grandes grupos de oferta: hotelera, extrahotelera y rural, así como su evolución. Para conseguirlo, se utilizaron tanto el análisis de clúster y de valor atípico (LISA) como el análisis de puntos calientes y fríos (Gl* de Getis-Ord). Ambas técnicas corroboran que los cambios detectados en la oferta de alojamientos siguen pautas similares y demuestran que la proximidad espacial no tiene un carácter espurio. Todo ello confirma el contrapuesto desarrollo turístico de Extremadura.

Palabras clave: Anselin Local Moran's I; Extremadura; geoestadística; Gi* de Getis-Ord.

\begin{abstract}
The quick increase in the supply of accommodation in Extremadura has not been matched by a corresponding increase in the number of visitors and overnight stays, a fact that has led to a significant mismatch between supply and demand. The increase in the supply has distributed unevenly over the territory. Thus, this paper aims to determine the distribution patterns detected within the three major supply groups: hotel, extra-hotel and rural as well as their evolution. In order to do that, cluster and outlier analysis (LISA) in addition to hot and cold spots analysis (Getis-Ord $\mathrm{GI}^{*}$ ) have been used. Both techniques confirm that the changes observed in the supply of accommodation follow similar patterns and show that the spatial proximity is not spurious. All this corroborates the existence of an antagonistic tourism development in Extremadura.
\end{abstract}

Key words: Anselin Local Moran's I; Extremadura; geostatistics; Getis-Ord Gi*.

Agradecimientos: La presente publicación ha sido posible gracias a la financiación recibida por el grupo de investigación en Desarrollo Sostenible y Planificación Territorial procedente del FEDER y de la Junta de Extremadura, a través de la ayuda GR15121.

\title{
1 Introducción
}

Extremadura dispone de grandes atractivos que actúan como reclamo para un número creciente de turistas. Cabe destacar el volumen de visitantes que reciben ciudades como Cáceres (Rengifo et al. 2015) y Mérida, ambas declaradas Ciudades Patrimonio de la Humanidad por la UNESCO, a las que se unen otras como Guadalupe, cuyo Monasterio también se encuentra protegido, Trujillo, Plasencia, o Badajoz, así como numerosas poblaciones dispersas por todo el territorio (Hervás, Zafra, Jerez de los Caballeros, Coria, Olivenza, etc.). Son enclaves llenos de encanto que se complementan con numerosos paisajes privilegiados, protegidos a través de la Ley 8/1998, de 26 de junio, de Conservación de la Naturaleza y Espacios Naturales de Extremadura, modificada por la Ley 9/2006, de 23 de diciembre. Estos espacios protegidos se extienden por $1257787 \mathrm{Ha}$, si bien, destacan por su enorme valor y potencial turístico, el Parque Nacional de Monfragüe o las 
Reservas de la Biosfera de Monfragüe y de Tajo-Tejo Internacional. También protegido por la UNESCO se halla el Geoparque Villuercas-lbores-Jara (Pulido et al., 2011).

Éstos y otros atractivos han facilitado la proliferación de la planta de hospedaje, circunstancia que ha propiciado que el turismo pase a ser una actividad interesante para la economía regional, ya que el sector turístico tiene una contribución notable al Producto Interior Bruto (PIB). Esto se observa en buena parte de las comunidades autónomas de España, si bien, en Extremadura su peso es sensiblemente inferior al de otros entornos, tanto en términos porcentuales como netos. En 2014, su contribución era aproximadamente del $5 \%$ del PIB regional, lo que se traduce en una riqueza de poco más de 861 millones de euros, cifra que apenas representa crecimiento respecto a 2009, cuando se obtenían alrededor de 860 millones $€$ (INE, 2015).

Debido al débil aumento neto de la contribución del sector de la hostelería a la riqueza de Extremadura, el Ejecutivo Regional se planteó duplicar esta cifra en el horizonte de 2020, si bien, esta aspiración puede parecer demasiado pretenciosa, a la vez que precisaría una planificación capaz de maximizar la capacidad de atracción. Este deseo supondría apostar por políticas conjuntas, que abarquen territorios amplios y próximos, sobre todo en aquellos espacios que disponen de menor capacidad de atracción, pero que pueden beneficiarse de los núcleos cercanos que hayan podido desarrollar la actividad turística.

A pesar del leve incremento de ingresos reflejado en la Contabilidad Regional de España (INE), no se puede negar que la situación actual del sector turístico extremeño no es tan buena como la experimentada unos años antes. Se han generado importantes desajustes, originados por la crisis económica y financiera que ha afectado a la región desde 2007 y 2009, respectivamente, tal como se deduce del hecho constatado de la cifra de viajeros y pernoctaciones registrados en Extremadura, comparado con la disponibilidad de plazas en todos los tipos de alojamiento. Si se fija como referencia los datos recogidos por el Instituto Nacional de Estadística (INE), en las encuestas de ocupación referidas a los diferentes tipos de alojamiento, se constała que en 2007 las plazas ofertadas alcanzaban como promedio las 27 488, mientras que en 2014, su número se elevaba un $26 \%$, hasta alcanzar un total de 34 614. En cambio, según esta misma fuente, los viajeros apenas aumentaron un $5 \%$ entre ambos años (1 409994 frente a 1481684) y las pernoctaciones se mantuvieron prácticamente igual (2 706402 por 2705 759). Por su parte, si se profundiza un poco más en los datos correspondientes a los diferentes tipos de oferta, se observa cómo los cambios son preocupantes en sectores clave, como el hotelero y el rural, debido a que el aumento de plazas ha sido más significativo que el de viajeros o de pernoctaciones, tal y como se recoge en la Tabla 1. La evolución que presentan las ratios entre pernoctaciones y plazas, muestran descensos de hasta un $24 \%$ en el caso del sector hotelero y de un $16 \%$ en el rural, curiosamente los dos tipos de alojamiento que captan más turistas y donde se produce mayor número de 
pernoctaciones. Además, el panorama del resto de variedades de alojamientos tiene una tendencia similar.

La localización de la oferta de alojamientos turísticos, sea cual fuere su modalidad, presenta un notable interés para determinar las políticas turísticas más adecuadas a cada espacio o para facilitar la planificación eficaz y coherente de la actividad. El beneficio que reporta el análisis territorial de los establecimientos turísticos vinculados al alojamiento viene dado por la trascendencia que tiene determinar el nivel de concentración o aislamiento que presenta. Además, resulta interesante conocer cómo ha evolucionado dicha implantación.

En nuestro estudio se apuesta por analizar no sólo la distribución per se de la oferta alojativa, sino también por la utilización del carácter de vecindad, de tanta trascendencia en todo hecho vinculado espacialmente. Ello se entiende si se considera que buena parte del territorio tiene notables afinidades en zonas muy concretas, a veces coincidentes con comarcas naturales y de dilatada trayectoria histórica (Las Hurdes, La Vera, El Jerte, etc.). Ello conlleva la posibilidad de generar productos turísticos específicos, vinculados siempre a la presencia de atractivos turísticos.

Tabla 1. Inadaptación entre oferta y demanda en Extremadura

\begin{tabular}{|l|c|c|c|c|c|c|c|c|c|}
\hline & \multicolumn{2}{|c|}{ Plazas } & \multicolumn{2}{c|}{ Viajeros } & \multicolumn{2}{c|}{ Pernoctaciones } & \multicolumn{2}{c|}{ Pernoctaciones / Plaza } \\
\cline { 2 - 11 } & 2007 & 2014 & 2007 & 2014 & 2007 & 2014 & 2007 & 2014 & $\%$ Var. \\
\hline Hoteles & 16361 & 19558 & 1170045 & 1194636 & 2097557 & 2031233 & 128,2 & 103,9 & $-24,35$ \\
\hline Campamentos & 6105 & 7056 & 104986 & 112922 & 282756 & 284953 & 46,3 & 40,4 & $-5,93$ \\
\hline Apartamentos & 709 & 1554 & 17156 & 39050 & 51938 & 84585 & 73,2 & 54,4 & $-18,77$ \\
\hline Turismo rural & 4313 & 6448 & 117807 & 135076 & 274151 & 304988 & 63,6 & 47,3 & $-16,26$ \\
\hline
\end{tabular}

Fuente: INE

Como ha quedado patente, la consecuencia inmediata de la crisis económica y financiera se vio reflejada de forma directa sobre el volumen de viajeros y de pernoctaciones registrados en los establecimientos de la Comunidad Autónoma de Extremadura, ya que han dejado de crecer de forma sostenida para estancarse e incluso retroceder, dependiendo del parámetro analizado.

A pesar de ello, aunque las dificultades económicas por las que atraviesa el país son las responsables directas de la merma de turistas y de pernoctaciones, no es la única causa que provoca dificultades dentro del sector. Posiblemente sea consecuencia de una política mal diseñada, que ha favorecido la instalación de nuevos alojamientos, pese a que la demanda no se incrementaba, cuando lo realmente necesario era apostar por políticas turísticas conducentes a la generación y consolidación de productos turísticos, sin recurrir en exceso a las políticas expansivas de creación de alojamientos.

Es difícil comprender el desarrollo de la actividad turística, debido a las múltiples interrelaciones que se establecen entre factores condicionantes y facilitadores así como entre los territorios o 
tipologías de turismo dominantes en espacios turísticos diferenciados. No obstante, en la literatura se observan ciertas tendencias que ofrecen explicaciones variadas, a la vez que se incrementan los estudios del sistema turístico realizados desde enfoques y perspectivas diferentes. Entre éstas destaca la postura ambiental (Buckley, 2011), la geográfica (Hall et al., 2014), la antropológica (Smith, 2012) y, por supuesto, la económica (Mohammed et al. 2015). En la mayor parte de los casos, cada uno de estos enfoques ha sido tratado generalmente de forma diferenciada y aislada, pese a que todos ellos interactúan en múltiples dimensiones. Por lo tanto, se puede pensar que están interrelacionados y no pueden ofrecer una visión global, a no ser que se analicen de forma conjunta e interconectada (Santana, 2003). No en vano, el turismo se sustenta en el territorio y éste se caracteriza por una interrelación entre numerosos factores y elementos. En él, vive una sociedad y se encuentran los recursos que actúan como reclamo para la demanda, y existen numerosas infraestructuras favorecedoras del desarrollo turístico, a la vez que todo ello se pone en valor dentro del sistema económico.

Existen múltiples causas que favorecen el avance del turismo y actúan normalmente de forma complementaria, interactuando y retroalimentándose, si bien, es cierto que el factor fundamental que explica el desarrollo de la actividad turística en un territorio es la presencia de recursos y su aprovechamiento (Cawley et al., 2008; Hall, 2011). Sin embargo, en ocasiones, se presupone que el patrimonio cultural o natural tiene un atractivo muy superior al real, pues la capacidad de atracción se ve modificada por numerosos parámetros externos como la accesibilidad, la propia oferta de alojamientos o complementaria, etc. Por ello, cada vez resulta más frecuente encontrar estudios donde prima la evaluación de su potencial como recurso turístico partiendo de los atractivos (Zhou et al., 2015), aunque también intervienen aspectos como la imagen del destino (Baloblu et al., 1999; Stylidis et al., 2014) y, por supuesto, ciertos parámetros geográficos (Hall et al., 2009; Sánchez et al., 2013) como el grado de concentración de atractivos en una zona, la distancia entre los centros emisores y receptores, la accesibilidad, etc.

Por su parte, la puesta en marcha de la actividad turística origina numerosos y variados impactos en el territorio donde se desarrolla (Mathieson et al., 1982), aunque existen estudios recientes que muestran los impactos positivos y negativos que genera la actividad en el turismo mundial (Archer et al., 2012). No obstante, la literatura se ha centrado en el análisis de los impactos económicos del turismo (Song et al., 2012), ya sea en el ámbito microeconómico o macroeconómico. Por el contario, existen menos referencias cuando se refieren a los impactos que soporta el medio y la sociedad a la que afecta. (Rutty et al., 2015).

Con el fin de minimizar los impactos negativos, e incrementar los positivos, se han puesto en marcha múltiples iniciativas de planificación turística. Ésta se erige como herramienta clave para el desarrollo armónico de la actividad, preservando el medio, vinculando a la sociedad residente, y obteniendo un rendimiento económico. Existen numerosas referencias bibliográficas sobre la 
planificación turística (Gunn, 1988; Inskeep, 1991; Simmons, 1994; Dredge et al., 2015), muy desarrolladas en la década de los noventa, si bien, continúan las aportaciones sobre esta temática (Jordan, 2015; Morpeth et al., 2015), aprovechando las constantes mejoras de las técnicas y herramientas analíticas.

Así mismo, existen numerosas metodologías para planificar el territorio, aunque pueden sintetizarse en cuatro grandes categorías (Antón et al., 2011):

a. las técnicas encargadas de recopilar opiniones e identificar los temas clave de la planificación territorial de un espacio, entre las que destacan el análisis DAFO y el método DELPHI.

b. las técnicas que se ocupan de obtener diagnósticos sintéticos y claros sobre la situación en que se encuentra el sistema turístico del lugar objeto de planificación.

c. las técnicas prospectivas cuyo cometido principal es promover escenarios de futuro para el ver el posible comportamiento del modelo turístico.

d. las técnicas de participación pública.

Aparte de estas técnicas y metodologías de planificación turística, conviene reseñar las herramientas disponibles para llevar a cabo la siempre difícil tarea de planificar (Ritchie et al., 2005). Existen muchos instrumentos para realizar una buena planificación; sin embargo, a veces adolecen del carácter espacial básico en el concepto del propio sistema turístico. Por este motivo, desde hace varias décadas se utilizan los Sistemas de Información Geográfica (SIG) como una herramienta básica para el análisis territorial del turismo en casi cualquiera de sus facetas, dada la capacidad analítica que posee, tanto para hacer consultas, como para abordar análisis espaciales de diferente tipo. Sin embargo, este tipo de técnicas presentaba una carencia clave, el análisis geoestadístico, por lo que era preciso recurrir a otro tipo de metodologías, de difícil manejo y comprensión, para aplicar variados algoritmos y proceder a su cartografiado.

Afortunadamente, los programas informáticos comerciales y algunos de código abierto han comenzado a incorporar este tipo de técnicas de análisis, ofreciendo numerosas posibilidades de trabajo y ensayos a los investigadores. Fiel reflejo de esa situación es la cantidad de literatura reciente (Polo y Chica, 2015; Sarrión et al., 2015; Fang et al., 2015; García et al., 2015) que apuesta por aplicaciones relacionadas con múltiples aspectos turísticos donde la estadística espacial tiene un papel destacado.

Considerando lo anterior, en la investigación se propone un análisis geoestadístico que permita evaluar si existen patrones de distribución ocultos o si se puede mejorar la actividad recurriendo al criterio de vecindad. 


\section{Metodología}

La realización de esta investigación se ha llevado a cabo con el software ArcGis 10.3, ya que facilita la ejecución de análisis estadísticos tomando el soporte territorial como elemento destacado.

Tanto esta herramienta como el conjunto de técnicas geoestadísticas se aplican para resolver los más variados problemas de la sociedad actual, desde el urbanismo a la localización de inversiones o de poblaciones en riesgo de exclusión, pasando obviamente por el turismo. En este último aspecto, destacan los estudios vinculados al análisis de los impactos generados por la actividad turística (Mason, 2015); las aplicaciones conducentes a la detección de tipologías de turismo y comportamientos de los turistas (Grinberger et al., 2014); también se utilizan para evaluar la centralidad espacial mediante análisis de redes (Lee et al., 2013); para determinar el potencial turístico que posee el territorio o los recursos (Rutherford et al., 2015); o para abordar de forma integral la gestión del turismo (Ofobruku et al., 2013), etc.

La geoestadística, como técnica de análisis, ha sido definida como una rama de la estadística aplicada que se especializa en el análisis y el modelado de la variabilidad espacial de un parámetro (Díaz, 2002). Su objeto de estudio es el análisis y la predicción de fenómenos dispersos, tanto en el espacio como en el tiempo.

El software elegido destaca precisamente por la inclusión de un módulo específico dedicado a la estadística espacial. En concreto, a efectos de este estudio, nos centramos en el análisis de agrupaciones que sirven para identificar puntos o zonas que son estadísticamente significativos. Este análisis de patrones espaciales es muy variado y abarca fundamentalmente:

a. El análisis de agrupación o de valores atípicos, representados por los algoritmos de Anselin Local Moran's I (LISA).

b. El análisis de punto caliente utilizando los criterios definidos por Getis-Ord en su estadístico Gi* .

c. El análisis de agrupaciones, si bien dada su complejidad no puede ser abordado en este estudio.

Este conjunto de técnicas permite detectar lugares de concentración, de dispersión o atípicos, así como las agrupaciones más significativas que ha sufrido la oferta de alojamientos en Extremadura a lo largo de la última década.

El objetivo que se plantea en este trabajo consiste en aplicar técnicas de mapping clúster para detectar la presencia de zonas donde los cambios en la oferta de alojamientos han sido mayores en tres periodos diferentes, 2004, 2009 y 2014. Se han seleccionado estos años porque marcan la situación existente antes de la crisis, durante los primeros años de la misma y, finalmente, cuando el escenario tiende a normalizarse. Los cambios se estudiarán durante una década para ver su evolución en los alojamientos hoteleros, extrahoteleros y rurales. Para alcanzar dicho objetivo se 
apuesta por procesos que permitan descubrir patrones de asociación espacial, tomando como referencia la unidad administrativa de la capital de término municipal, si bien, en aras de mayor claridad en la exposición cartográfica, los resultados se han implementado en el término municipal. Además, conviene clarificar que si en 2004 existían 382 municipios, se ha optado por considerar los 388 actuales, ya que los nuevos municipios que se han segregado no disponían anteriormente de la oferta turística analizada y en 2014, mantienen esa carencia.

Para la elaboración de esta investigación se parte de la información suministrada por la Dirección General de Turismo de la Junta de Extremadura.

El trabajo se organiza en cinco apartados diferenciados. El primero trata de introducir al lector en la problemática que tiene el turismo en la comunidad de Extremadura, debido básicamente al estancamiento de los ingresos por el turismo y al seguimiento de políticas de desarrollo turístico expansivo, así como a la justificación de la geoestadística como modelo de análisis. El segundo muestra la evolución del alojamiento y su distribución en el contexto territorial analizado, lo que permitirá entender los desajustes territoriales que aparecen en la oferta de alojamientos. En el tercero, se explica la metodología propuesta para alcanzar los objetivos planteados, a pesar de que existe profusa literatura científica donde se puede encontrar el funcionamiento de los algoritmos de análisis propuestos. El cuarto capítulo explica los resultados principales que se obtienen tras la aplicación metodológica; mientras tanto, el quinto se centra en la detección de los cambios que se han producido en el contexto territorial. Por último, en el sexto, se exponen las conclusiones principales que han sido extraídas.

El análisis del sector turístico posee una fuerte componente espacial, de tal forma que buena parte de las variables turísticas están condicionadas por los valores que toman de lugares próximos al destino, por lo que parece oportuno aplicar técnicas de autocorrelación espacial, clústers espaciales, etc. (Sánchez, 2008). Estas técnicas, pueden verse complementadas con otras, tal vez más complejas, orientadas hacia la inferencia, hacia el cálculo predictivo, como sucede con la regresión geográficamente ponderada (GWR) (Sánchez et al., 2013)

Los procesos agrupados en el conjunto de la estadística espacial ofrecen la posibilidad de realizar un análisis pormenorizado del territorio, recurriendo para ello a criterios de proximidad, de colindancia o de vecindad. Todos ellos consideran las interrelaciones entre los casos analizados en un contexto variable, determinado por el espacio próximo como un elemento destacable. Estas relaciones espaciales fueron reconocidas ya mediante la denominada primera Ley de Tobler, dentro de la disciplina geográfica, al enunciar éste que todo estaba relacionado con cualquier cosa, a la vez que precisaba que lo cercano estaba más relacionado que lo distante (Tobler, 1970). Esta peculiaridad de análisis integrado en el contexto territorial más inmediato resulta clave para entender la generación de áreas que concentren numerosa oferta alojativa o, por el contrario, escasa. 
Desde esta óptica, el análisis estadístico que se hace en el contexto del sector turístico puede enriquecerse notablemente con la aplicación de los criterios espaciales que aporta la geoestadística. Así, la distribución territorial del turismo no es homogénea ni presenta síntomas de aleatoriedad, sigue unas pautas, a veces difusas, pero visibles cuando se analizan desde la perspectiva geográfica. La ubicación de la oferta en el territorio parte de la existencia de recursos que permitan sustentar la actividad, pero también de otros aspectos que, en gran medida, han sido definidos como factores de localización, tal como han manifestado variados autores (Gunn, 1988; Ritchie et al., 2005; Antón et al., 2011; Sánchez, et al. 2013).

La adopción e integración de las dimensiones geográficas al Turismo ha puesto énfasis en la detección de patrones generales de distribución, aplicando para ello tanto consideraciones teóricas como empíricas, y cuya base metodológica ha partido de la aplicación de los más variados métodos de análisis locacionales del turismo (Yokeno, 1968; Haggett, 1976) hasta llegar a nuestros días, en los que se hace una utilización profusa de los análisis geoestadísticos (García-Palomares et al., 2015; Fang et al., 2015). Por este motivo, se propone una comparación entre diferentes técnicas orientadas a descubrir agrupaciones o concentraciones en la distribución de la oferta de alojamientos, así como la evolución que presenta su implantación, como una forma de comprobar las áreas que pueden presentar algún tipo de problema. Tras su aplicación, se comprobará cuál o cuáles permiten obtener mejores resultados para el análisis de la distribución espacio-temporal. Las técnicas propuestas para el análisis comparativo parten de las herramientas de asignación de clústers presentes en el módulo de estadística espacial del software ArcGIS 10.3 (ESRI, 2015).

En líneas generales, este tipo de técnicas de análisis de agrupaciones puede resultar muy útil para determinar si existen grupos de municipios bien definidos y caracterizados por disponer de oferta de alojamiento con características similares, tanto al considerar el volumen de plazas, como al tener en cuenta las tipologías de alojamiento o cualquier otra variable que puede localizarse sobre el territorio. De acuerdo con ello, se pueden precisar las áreas donde existe una mayor concentración de oferta de alojamientos, lo que daría lugar a la generación de un punto caliente o, visto de otra forma, una posible sobreoferta. Al mismo tiempo, también posibilitaría detectar grupos de municipios en los que el volumen de plazas de alojamiento ofrecidas es escaso, con lo que se generaría un punto frío. Además, también pueden existir territorios que presenten anomalías en los registros de oferta. Sendos aspectos relativos a los puntos calientes, puntos fríos o anomalías, servirán para inferir las posibles causas que los originan.

Como complemento a este análisis territorial, se propone la utilización de un enfoque temporal, que sirva para determinar el comportamiento de la oferta de alojamientos a lo largo de la última década, para lo que se tomarán como referencia los datos relativos a las tres grandes tipologías de alojamiento (hoteleros, extrahoteleros y rurales) durante tres años diferentes: 2004, 2009 y 2014 
En las diferentes técnicas estadísticas que se usarán, se ha optado por aplicar una relación espacial que siga el criterio de distancia inversa al cuadrado, puesto que se estima que la influencia entre los elementos próximos disminuye rápidamente a medida que se produce un alejamiento del punto analizado. Además, para dar mayor uniformidad a los cálculos, se utilizará el término municipal como entidad de análisis.

\subsection{Anselin Local Moran's I}

El análisis de clúster y de valor atípico (LISA) tiene como objetivo fundamental determinar el nivel de agrupación territorial que existe en los valores registrados por una variable dada para el conjunto de observaciones. Toma como elemento preceptivo no sólo el valor de la variable, sino también el criterio de vecindad. Todo ello redunda en la obtención de 5 grupos o clases bien diferenciados, que toman como base para su cálculo tanto el criterio numérico como el territorial.

Los conjuntos definidos mediante esta técnica de análisis territorial representan 2 niveles de agrupación diferentes. El primero mostraría una zona donde se producen concentraciones de valores elevados, o bajos en la variable analizada, dando lugar a puntos calientes $(\mathrm{HH})$ o fríos (LL). El segundo, en cambio, identificaría aquellos puntos con comportamientos atípicos respecto al entorno más inmediato, e igualmente ofrecen dos configuraciones, una donde el valor anormal muestra un valor claramente más elevado que la zona próxima $(\mathrm{HL})$, y otra que representa un valor anormalmente bajo en el contexto territorial en el que se ubica $(\mathrm{LH})$. Aparte de estas 4 clases, existe una última agrupación para todas aquellas zonas en las que no se detecta un patrón de relación claro. Se trataría de las zonas que obtienen valores no estadísticamente significativos.

La formulación matemática que define este estadístico es compleja, tal como puede verse en la siguiente ecuación (Anselin, 1995):

$$
I_{i}=\frac{x_{i}-\bar{X}}{S_{i}^{2}} \sum_{j=1, j \neq i}^{n} w_{i, j}\left(x_{i}-\bar{X}\right)
$$

donde $x_{i}$ es el atributo analizado para la geometría $i, \bar{X}$ es el promedio que se corresponde con el atributo, $W_{i, j}$ es el peso espacial entre la función de la geometría $i$ y $j$. Por su parte, para determinar el componente $S_{i}^{2}$ se recurre a la siguiente ecuación:

$$
S_{i}^{2}=\frac{\sum_{j=1, j \neq i}^{n} w_{i, j}}{n-1}-\bar{X}^{2}
$$

siendo $n$ el número total de geometrías consideradas.

Como el resultado de este estadístico se sintetiza en la obtención de z (desviación estándar) y p (probabilidad asociada al intervalo de confianza), conviene reseñar que se obtiene mediante: 
$Z I_{i}=\frac{I_{i}-E\left[I_{i}\right]}{\sqrt{V\left[I_{i}\right]}}$

donde $E\left[I_{i}\right]=-\frac{\sum_{j=1, j \neq i}^{n} W i j}{n-1}$ y $V\left(I_{i}\right)=E\left[I_{i}^{2}\right]-\mathrm{E}\left[I_{i}\right]^{2}$.

Para la obtención de estos índices, se han tomado como referencia las 388 capitales municipales con que cuenta Extremadura y, tras diferentes ensayos, se ha optado por un tipo de relación de distancia, en este caso, euclidiana y bajo el criterio de distancia inversa al cuadrado, de tal modo que se estima que la capacidad de relación decae de forma rápida con la distancia. Pese a ello, se ha utilizado una distancia nada desdeñable de $12 \mathrm{~km}$, partiendo de la base de que el propio software recomendaba 11,8km considerando la especial distribución de los puntos de observación, los núcleos de población.

Por lo que respecta a las variables utilizadas, cabe señalar que se ha optado por centrarse en la evolución que han experimentado entre 2004 y 2014 las plazas en los tres tipos de alojamiento seleccionados (hotelero, extrahotelero y rural), así como la capacidad total de la oferta. El hecho de utilizar agrupaciones de oferta se debe a que un nivel de desagregación menor en el caso de Extremadura implica que no se disponga de información suficiente para la realización de los cálculos de manera fiable y robusta.

\subsection{Gi* de Getis-Ord}

El índice Gi* diseñado por Getis y Ord mide el grado de asociación que resulta de la concentración ponderada de puntos, donde el valor de éste incluye a todos los demás en la distancia determinada para su cálculo (Getis y Ord, 1992). Calcula los puntos calientes y a los puntos fríos. Los primeros se forman cuando en un entorno de vecindad, los valores que alcanza la variable analizada son altos; por el contrario, los segundos se originan cuando los valores son bajos.

Los resultados que ofrece esta técnica se muestran en forma de puntuaciones $z$ y p, desviaciones estándar y probabilidades respectivamente. La combinación de sendos parámetros permite detectar la presencia de puntos calientes o fríos, siempre que posea una significación estadística al presentar valores próximos a los alcanzados en el entorno inmediato.

El cálculo de esta técnica se define mediante la siguiente ecuación:

$$
G_{i}^{*}=\frac{\sum_{j=1}^{n} w_{i, j} x_{j}-\bar{X} \sum_{j=1}^{n} w_{i, j}}{S \sqrt{\frac{\left[n \sum_{j=1}^{n} w_{i, j}^{2}-\left(\sum_{j=1}^{n} w_{i, j}\right)^{2}\right]}{n-1}}}
$$


En dicha fórmula $x_{j}$ representa el valor que tiene el atributo analizado en la geometría j. Por su parte, $w_{i j}$ es el peso territorial que corresponde a la característica $i$ y $j$, así como $n$ es igual al número total de geometrías.

Para su cálculo se ha optado por aplicar los mismos parámetros que en la técnica anterior, con el objetivo de comprobar y comparar los resultados de ambas. Así, como entidad territorial se toman los núcleos de población principal de cada municipio y como variables analizadas los tres grandes grupos de tipo de oferta. De igual modo, tras numerosos ensayos, se han definido las relaciones espaciales a través de la distancia euclidiana inversa al cuadrado.

\section{Resultados y discusión}

La oferta de alojamientos existente en Extremadura presenta una distribución dicotómica. Por una parte, se encuentran los núcleos urbanos, en los que se concentran numerosos establecimientos hoteleros. Por otra, existen zonas rurales, en las que el predominio de oferta de alojamientos se centra en los establecimientos rurales y extrahoteleros, si bien, estos últimos aparecen también en las ciudades. El entramado territorial que configura la oferta se completa con otros núcleos que carecen de establecimientos. Se trata, por tanto, de una situación complicada, que da lugar a un complejo entramado de zonas con orientaciones turísticas diferenciadas, a lo que se une un desigual desarrollo turístico, aparte de la evolución lógica que se produce desde 2004.

Si se analiza conjuntamente la distribución territorial de esta oferta de alojamientos a lo largo de la última década, se detecta un aumento significativo, no sólo en el volumen de establecimientos y plazas, sino también un creciente número de núcleos que tratan de desarrollar esta actividad. Así, en 2004, existían 176 municipios que disponían de algún tipo de alojamiento, mientras que en 2014 eran 284 municipios. Es decir, en apenas una década, la actividad turística trata de abrirse camino en buena parte de los municipios extremeños.

Esta situación, generalizada para el conjunto de Extremadura, encubre ciertas diferencias cuando se analiza con mayor detalle territorial. De hecho, un reducido número de municipios concentra buena parte de los establecimientos. Se trata de las principales ciudades (Cáceres, Mérida, Badajoz o Plasencia) y cabeceras comarcales (Trujillo, Zafra, Valencia de Alcántara o Almendralejo), así como de otros núcleos que disponen de algún tipo de recurso muy específico. Tal es el caso de Baños de Montemayor, Jarandilla de la Vera, Hervás o Guadalupe, a los que también se pueden añadir otros como Navaconcejo, Monesterio, Caminomorisco o Alange.

En buena parte de los casos, la presencia de atractivos turísticos, sea del tipo que fueren, prevalece como criterio principal para la instalación de oferta. Prueba de ello, es el hecho de que entre los núcleos con mayor número de plazas de alojamiento se encuentren tres tipologías distintas. En primer lugar, aquellos que aglutinan el mayor atractivo cultural (refrendado por ser 
Patrimonio de la Humanidad por la UNESCO) (Rengifo et al., 2015). En segundo lugar, los que disponen de los principales atractivos que demanda el turista rural, como las zonas de montaña, las piscinas naturales o algunos espacios naturales (Sánchez, 1999; Sánchez et al., 2001; Sánchez et al., 2013) y, finalmente, los que disponen de un recurso específico, como pueden ser los balnearios.

El análisis específico de cada tipología de oferta, ya sea hotelera, extrahotelera o rural, muestra una vez más que el territorio extremeño tiene vocaciones turísticas diferenciadas, tal como se recoge en la Tabla 2 y se representa en la Figura 1. Las principales ciudades cuentan con abundante oferta hotelera, si bien su volumen depende básicamente del potencial turístico que aglutina. Así, se observa cómo Cáceres o Mérida, ambas Patrimonio de la Humanidad, disponen de un número considerable de plazas hoteleras, al igual que Badajoz, ya que superan las 2000 plazas en este tipo de alojamiento. A notable distancia se encuentran Trujillo, Plasencia, Zafra o Almendralejo, con un rango de plazas en este tipo de alojamientos que abarca desde las 1041 hasta las 606 . A nivel territorial existe una distribución muy variada y sostenida a lo largo de la década analizada.

Tabla 2. Distribución de plazas de alojamiento según tipologías y años en municipios turísticos

\begin{tabular}{|c|c|c|c|c|c|c|c|c|c|c|c|c|}
\hline \multirow[b]{2}{*}{ Municipios } & \multicolumn{3}{|c|}{ Plazas totales } & \multicolumn{3}{|c|}{ Plazas hoteleras } & \multicolumn{3}{|c|}{ Plazas extrahoteleras } & \multicolumn{3}{|c|}{ Plazas rurales } \\
\hline & 2004 & 2009 & 2014 & 2004 & 2009 & 2014 & 2004 & 2009 & 2014 & 2004 & 2009 & 2014 \\
\hline Cáceres & 2074 & 2.127 & 3321 & 1536 & 1.536 & 2578 & 538 & 591 & 713 & 0 & 0 & 30 \\
\hline Mérida & 1847 & 1913 & 2586 & 1492 & 1558 & 2068 & 346 & 346 & 509 & 9 & 9 & 9 \\
\hline Badajoz & 1571 & 1789 & 2274 & 1571 & 1789 & 2274 & 0 & 0 & 0 & 0 & 0 & 0 \\
\hline Jarandilla de la Vera & 1334 & 1348 & 1591 & 121 & 121 & 254 & 1089 & 1089 & 1093 & 124 & 138 & 244 \\
\hline Plasencia & 1292 & 1316 & 1433 & 876 & 876 & 980 & 410 & 434 & 436 & 6 & 6 & 17 \\
\hline Baños de Montemayor & 1022 & 1162 & 1411 & 296 & 296 & 500 & 726 & 750 & 750 & 0 & 116 & 161 \\
\hline Trujillo & 924 & 972 & 1252 & 890 & 890 & 1041 & 0 & 36 & 78 & 34 & 46 & 133 \\
\hline Hervás & 969 & 1110 & 1224 & 112 & 112 & 174 & 781 & 805 & 805 & 76 & 193 & 245 \\
\hline Losar de la Vera & 1037 & 1051 & 1121 & 189 & 189 & 201 & 848 & 848 & 858 & 0 & 14 & 62 \\
\hline Jerte & 709 & 845 & 878 & 121 & 121 & 121 & 473 & 483 & 492 & 115 & 241 & 265 \\
\hline Guadalupe & 681 & 700 & 770 & 409 & 409 & 422 & 222 & 222 & 250 & 50 & 69 & 98 \\
\hline Zafra & 540 & 562 & 746 & 512 & 534 & 718 & 0 & 0 & 0 & 28 & 28 & 28 \\
\hline Madrigal de la Vera & 572 & 585 & 697 & 80 & 80 & 100 & 480 & 480 & 480 & 12 & 25 & 117 \\
\hline Navaconcejo & 453 & 558 & 690 & 0 & 0 & 0 & 369 & 381 & 386 & 84 & 177 & 304 \\
\hline Malpartida de Plasencia & 639 & 665 & 689 & 164 & 164 & 164 & 405 & 405 & 411 & 70 & 96 & 114 \\
\hline Monesterio & 473 & 491 & 670 & 146 & 146 & 305 & 327 & 334 & 334 & 0 & 11 & 31 \\
\hline Valencia de Alcántara & 465 & 521 & 627 & 116 & 116 & 116 & 269 & 281 & 281 & 80 & 124 & 230 \\
\hline Almendralejo & 372 & 372 & 606 & 372 & 372 & 606 & 0 & 0 & 0 & 0 & 0 & 0 \\
\hline Caminomorisco & 501 & 501 & 549 & 65 & 65 & 90 & 412 & 412 & 412 & 24 & 24 & 47 \\
\hline Alange & 99 & 180 & 540 & 99 & 99 & 446 & 0 & 0 & 0 & 0 & 81 & 94 \\
\hline
\end{tabular}

Fuente: Dirección General de Turismo (Junta de Extremadura) 
Una situación mucho más compleja caracteriza la distribución de la demanda extrahotelera, puesto que, en su mayor parte, se condiciona por la presencia de campamentos turísticos. Estos se instalan preferentemente en el ámbito rural, para favorecer ese contacto con la naturaleza, como lo demuestra el gran número de plazas que se oferta en numerosos núcleos del norte de Extremadura. Pese a ello, también hay un número de plazas abundantes en Cáceres o Mérida. A nivel territorial, esta oferta presenta mayor concentración espacial en la zona septentrional.

Figura 1. Evolución del número de plazas en alojamientos de Extremadura
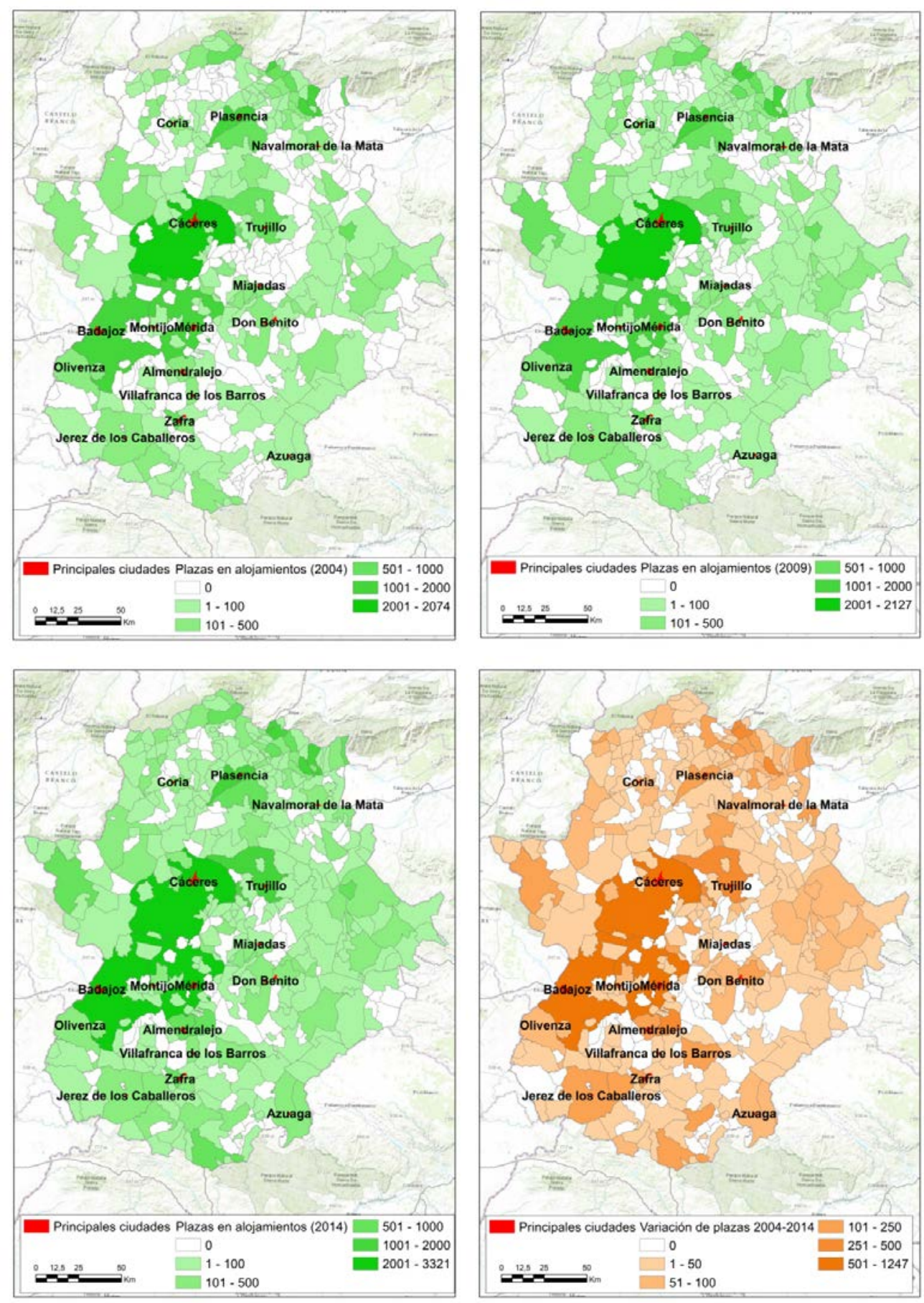

Fuente: elaboración propia 
Por lo que respecta a la oferta rural, conviene destacar que ha experimentado un crecimiento muy importante a lo largo de la última década, fruto de las cuantiosas inversiones realizadas mediante los programas LEADER y PRODER (Nieto y Gurría, 2008; Sánchez et al., 2013). A nivel territorial, se aprecia una distribución dispar sobre el territorio, si bien, un análisis detallado permite detectar patrones de distribución que, en buena parte de los casos, coinciden con las zonas montañosas, localizadas en el norte, centro y sur de Extremadura.

El panorama que refleja la evolución general en el número de plazas muestra que, desde el punto de vista de la oferta turística, las zonas más dinámicas se corresponden con las principales ciudades, a las que se unen zonas localizadas en el norte de la provincia de Cáceres. Mientras tanto, en el resto predominan aumentos de plazas sensiblemente inferiores y con desigual reparto territorial. Pese a la distribución, a veces errática, de las plazas de alojamiento, no se puede olvidar que, en varias zonas, existen ciertas pautas que reflejarían la importancia que tiene el criterio territorial en su distribución y, sobre todo, en su análisis y comprensión

Para determinar cómo ha sido la implantación de la oferta de alojamientos durante el periodo 20042014, se recurre a la utilización de dos parámetros geoestadísticos que miden los cambios producidos durante esa década. Sendos parámetros son el análisis de clúster y valor atípico I Anselin (LISA) y el análisis de punto caliente Gi* de Getis-Ord. Con estos dos algoritmos, ya explicados y fundamentados en la metodología, se busca detectar los lugares que presentan anomalías de comportamiento respecto de los circundantes y, también los espacios en que se han producido mayores incrementos en el volumen de oferta o, por el contrario, presentan deficiencias en este tipo de infraestructuras turísticas.

\subsection{Análisis clúster y valor atípico I Anselin}

La distribución territorial de la oferta de alojamientos ha variado a lo largo de los años, si bien, en líneas generales, sigue unas pautas que vienen marcadas, casi impuestas, por la presencia de los principales atractivos turísticos. No obstante, cuando se recurre a un análisis geoestadístico con el fin de considerar el comportamiento de un municipio con los que tiene más próximos, utilizando para ello el análisis de clúster y de valor atípico diseñado por Anselin, se aprecia la situación que reflejan diferentes entornos, tal como se muestra en la Figura 2 y en la Tabla 3. En este sentido, cabe destacar que en los tres años analizados, y que se toman como testigo para medir la evolución en la implantación de las plazas de alojamiento, hay una tendencia marcada de agrupación entre una parte considerable de los núcleos de las comarcas cacereñas de Valle del Jerte, La Vera y Valle del Ambroz, situadas al norte. Se trata de los municipios codificados como $\mathrm{HH}$. Es decir, en buena parte de estas zonas, si un municipio dispone de un volumen considerable de alojamientos, sus vecinos también. Esta situación nos permite corroborar la idea de que se puede hablar de destinos específicos y reconocidos, pues todos tienen en común el considerable 
poder de atracción que ejercen las zonas de montaña. Pese a ello, este análisis también refleja que hay otros municipios que, pese a disponer de los mismos atractivos, no han sido capaces de crear en torno a ellos una oferta de alojamientos adaptada a su potencial.

Al mismo tiempo, el dominio de los principales valores atípicos afecta a las principales ciudades de Extremadura (Badajoz, Cáceres, Mérida o Plasencia), a las que se une un núcleo de menor entidad demográfica como es Trujillo, motivado, sin lugar a dudas, por el enorme atractivo que posee, además de localizarse en una posición estratégica respecto de las principales vías de comunicación por carreteras de alta capacidad.

Figura 2. Análisis de clúster y valor atípico I Anselin
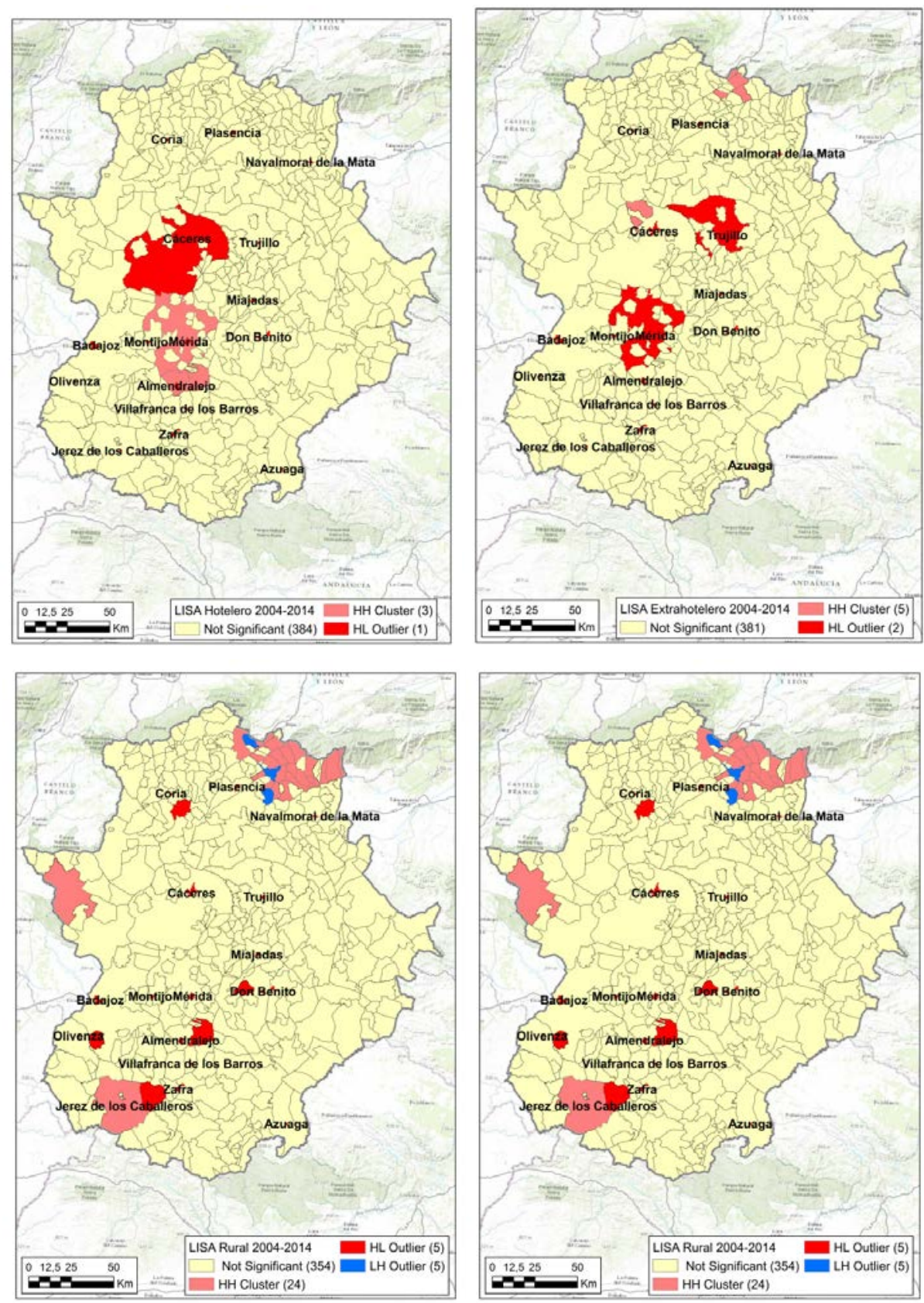

Fuente: elaboración propia 
Grosso modo esa es la situación genérica que afecta al conjunto de alojamientos durante los años objeto de análisis, aunque cuando se analizan los cambios registrados en el volumen de plazas en los tres grupos de oferta, la hotelera, la extrahotelera y la rural, se observan unos cambios que pueden ser indicios de la complejidad y heterogeneidad que presenta el sector turístico en Extremadura.

Así, la evolución del sector hotelero muestra una agrupación interesante en el eje Mérida-AlangeAlmendralejo, que configuran una zona de expansión notable de la actividad turística, por diferentes motivos, pero complementarios entre sí. De este modo, Mérida, uno de los principales destinos de turismo cultural de Extremadura, se complementa con Alange, que dispone de un balneario de primer nivel, además de un gran embalse en las proximidades, a la vez que en Almendralejo se concentra una de las zonas de mayor dinámica económica de Extremadura y principal sede del enoturismo de la región. Por su parte, Cáceres destaca como un valor atípico, ya que ningún municipio próximo (20 km en nuestros análisis) es tan dinámico.

Por su parte, la oferta extrahotelera también muestra dos zonas bien definidas, entre las que se encuentran las áreas muy diferenciadas del entorno (outlier) como Mérida y Trujillo. Al mismo tiempo, aparecen concentraciones de municipios con similares características en el norte de la provincia de Cáceres.

En lo que respecta a la evolución experimentada durante estos 10 años por la oferta de alojamientos rurales, cabe reseñar que existe un elevado grado de concentración en el norte de la provincia de Cáceres, a la vez que comienza a desarrollarse en otros espacios del sur de la provincia de Badajoz y en el oeste de Extremadura. Configuran el núcleo fundamental del turismo rural de Extremadura, erigidos como destinos de reconocido prestigio. Al mismo tiempo, también encuadrados en estas zonas septentrionales, aparecen otros núcleos que, pese a estar en el área de influencia de los anteriores, no disponen de alojamientos rurales y, por lo tanto, configuran una anomalía destacable. Este grupo (LH) dispone de un gran potencial de desarrollo turístico, si bien, dada su posición marginal, no forman parte del primer grupo. A sendos grupos se le añade el conformado por los valores atípicos, es decir, aquellos municipios que sí han tenido una evolución positiva en cuanto al número de plazas en alojamientos rurales, pero que sin embargo no se ven acompañados por el entorno inmediato.

De la aplicación de este análisis geoestadístico se deducen dos ideas fundamentales respecto de la evolución territorial experimentada por la oferta de alojamientos. La primera, determina que los mayores cambios producidos se concentran en las principales ciudades de rango turístico, así como en los núcleos del norte de la provincia de Cáceres y, la segunda, identifica al sector del alojamiento rural como uno de los más complejos, habida cuenta de la variedad de relaciones espaciales que se observa entre los municipios de Extremadura. Al mismo tiempo, es posible 
deducir que en buena parte del territorio existen relaciones espaciales poco definidas cuando se analiza la evolución de las plazas de alojamiento turístico desde 2004 hasta 2014.

Tabla 3. Análisis clúster y de valor atípico I Anselin (resumen de resultados)

\begin{tabular}{|c|c|c|c|c|c|c|}
\hline MUNICIPIO & $\begin{array}{l}\text { LISA } \\
2004\end{array}$ & $\begin{array}{l}\text { LISA } \\
2014\end{array}$ & $\begin{array}{l}\text { LISA } 2004 \\
\text { - } 2014\end{array}$ & $\begin{array}{l}\text { LISA HOT. } \\
\text { 2004-2014 }\end{array}$ & $\begin{array}{c}\text { LISA EXTRA- } \\
\text { HOT. } 2004- \\
2014\end{array}$ & $\begin{array}{c}\text { LISA } \\
\text { RURAL } \\
2004-2014\end{array}$ \\
\hline Abadía & & & & & & LH \\
\hline Alange & & & & $\mathrm{HH}$ & & $\mathrm{HL}$ \\
\hline Aldeanueva de la Vera & $\overline{\mathrm{HH}}$ & $\overline{\mathrm{HH}}$ & $\overline{\mathrm{HH}}$ & & & $\mathrm{HH}$ \\
\hline Aldeanueva del Camino & $\mathrm{HH}$ & & & & & $\mathrm{HH}$ \\
\hline Almendralejo & & & & $\mathrm{HH}$ & & \\
\hline Arroyomolinos de la Vera & & & & & & $\overline{\mathrm{HH}}$ \\
\hline Badajoz & & $\mathrm{HL}$ & $\mathrm{HL}$ & & & \\
\hline Baños de Montemayor & $\mathrm{HH}$ & $\mathrm{HH}$ & $\mathrm{HH}$ & & $\overline{\mathrm{HH}}$ & $\overline{\mathrm{HH}}$ \\
\hline Burguillos del Cerro & & & & & & $\mathrm{HL}$ \\
\hline Cabezuela del Valle & & & & & $\mathrm{HH}$ & $\mathrm{HH}$ \\
\hline Cabrero & & & & & & $\mathrm{LH}$ \\
\hline Cáceres & $\mathrm{HL}$ & $\mathrm{HL}$ & $\mathrm{HL}$ & $\mathrm{HL}$ & & \\
\hline \multicolumn{7}{|l|}{ Casar de Cáceres } \\
\hline Casas del Castañar & & & & & & $\overline{\mathrm{HH}}$ \\
\hline Casas del Monte & & & & & $\overline{\mathrm{HH}}$ & $\mathrm{HH}$ \\
\hline Cuacos de Yuste & $\mathrm{HH}$ & $\mathrm{HH}$ & $\mathrm{HH}$ & & & $\mathrm{HH}$ \\
\hline Garganta la Olla & & & & & & $\mathrm{HH}$ \\
\hline Hervás & $\mathrm{HH}$ & $\overline{\mathrm{HH}}$ & $\overline{\mathrm{HH}}$ & & $\overline{\mathrm{HH}}$ & $\mathrm{HH}$ \\
\hline Jaraíz de la Vera & & & & & & $\mathrm{HH}$ \\
\hline Jarandilla de la Vera & $\overline{\mathrm{HH}}$ & $\overline{\mathrm{HH}}$ & $\overline{\mathrm{HH}}$ & & & $\mathrm{HH}$ \\
\hline Jerez de los Caballeros & & & & & & $\mathrm{HH}$ \\
\hline Jerte & $\overline{\mathrm{HH}}$ & $\overline{\mathrm{HH}}$ & $\overline{\mathrm{HH}}$ & & & $\overline{\mathrm{HH}}$ \\
\hline Losar de la Vera & $\mathrm{HH}$ & $\overline{\mathrm{HH}}$ & $\overline{\mathrm{HH}}$ & & & $\overline{\mathrm{HH}}$ \\
\hline Madrigal de la Vera & & & & & & $\mathrm{HH}$ \\
\hline Medellín & & & & & & $\mathrm{HL}$ \\
\hline Mérida & $\mathrm{HL}$ & $\mathrm{HL}$ & $\mathrm{HL}$ & $\overline{\mathrm{HH}}$ & $\mathrm{HI}$ & \\
\hline Navaconcejo & $\mathrm{HH}$ & $\mathrm{HH}$ & $\mathrm{HH}$ & & & $\mathrm{HH}$ \\
\hline Pasarón de la Vera & & & & & & $\mathrm{HH}$ \\
\hline Piornal & & & & & & $\mathrm{LH}$ \\
\hline Plasencia & $\mathrm{HL}$ & $\mathrm{HL}$ & $\mathrm{HL}$ & & & ${ }_{-}$ \\
\hline Robledillo de la Vera & & & & & & $\overline{\mathrm{HH}}$ \\
\hline Tejeda de Tiétar & & & & & & $\mathrm{LH}$ \\
\hline Tornavacas & & & & & & $\mathrm{HH}$ \\
\hline Torrejoncillo & & & & & & $\mathrm{HL}$ \\
\hline Trujillo & $\mathrm{HL}$ & $\mathrm{HL}$ & $\mathrm{HL}$ & & $\mathrm{HI}$ & \\
\hline Valdastillas & & & & & & $\mathrm{LH}$ \\
\hline Valencia de Alcántara & & & & & & $\mathrm{HH}$ \\
\hline Valverde de la Vera & & & & & & $\mathrm{HH}$ \\
\hline Valverde de Leganés & & & & & & $\mathrm{HL}$ \\
\hline Villanueva de la Vera & & & & & & $\mathrm{HH}$ \\
\hline Zarza de Granadilla & & & & & & $\mathrm{HH}$ \\
\hline
\end{tabular}

Fuente: elaboración propia 


\subsection{Análisis de punto caliente Gi* de Getis-Ord}

Mediante la aplicación del análisis geoestadístico de Getis-Ord, se pretende determinar la presencia de zonas que reúnan condiciones homogéneas relativas a la evolución de la oferta de alojamientos presente en Extremadura, asimismo, durante el periodo 2004-2014, según se recoge en la Tabla 4 y se represent en la Figura 3.

El algoritmo utilizado por Getis-Ord busca detectar zonas calientes, definidas como aquellas en las que existe un sesgo positivo respecto a la desviación estándar global de la muestra, y áreas frías, donde el sesgo se produce de forma negativa. Es decir, se buscan asociaciones de municipios en las que el elemento clave sea definir si se ha producido un incremento o un descenso en el volumen de plazas registrado durante los años analizados.

De forma genérica, se detecta claramente que durante la década analizada no se han detectado puntos fríos. Predominan, por el contrario, los municipios en los que la desviación estándar obtenida y, consecuentemente, el índice $\mathrm{Gi}^{*}$ no tienen ninguna significación. A pesar de ello, esta misma técnica refleja dos concentraciones bastante llamativas a nivel territorial, ya que los tres valles más orientales del norte de la provincia de Cáceres, configuran un punto caliente destacado, aunque con distinto nivel de confianza. Esta misma circunstancia se detecta en núcleos cercanos al triángulo formado por las ciudades de Badajoz, Cáceres y Mérida.

De forma sintética, se puede decir que del total de los 388 municipios extremeños, en 337 el índice obtenido no es significativo. Por el contrario, en 51 se encuentran valores Gi* elevados o desviaciones estándar positivas, de lo que se deduce que conforman un punto caliente, tal como se indica en la Tabla 4. En concreto, en 22 nos encontramos con un punto caliente determinado con un $90 \%$ de confianza; en 14 de ellos, el nivel de confianza se eleva al $95 \%$ y, por último, en 15 el nivel de confianza es del $99 \%$. Como se puede apreciar, son valores que avalan la consideración de estas agrupaciones de municipios.

El análisis de los tres grupos de oferta de alojamientos, hotelera, extrahotelera y rural, refleja un comportamiento diferenciado entre unos entornos y otros, aunque en líneas generales, se corrobora la presencia predominante de puntos calientes sobre los fríos.

El sector hotelero presenta una evolución muy bien definida a lo largo de los años analizados, puesto que se configura un punto caliente muy bien delimitado entre las tres ciudades de mayor entidad demográfica, aparte del enorme atractivo turístico que tienen, sobre todo en el caso de Cáceres y Mérida. Este punto caliente, con porcentajes de confianza mínimos del $95 \%$, no ofrece ninguna duda sobre la disposición del mismo, algo comprensible si se tiene en cuenta que en esta área hay núcleos de gran aceptación por parte de la demanda turística. Estos núcleos, hacen que, poco a poco, el desarrollo turístico se extienda a las zonas próximas, no exentas de atractivos 
turísticos. En total, son 23 los municipios que configuran este punto caliente; mientras tanto, los 365 municipios restantes no alcanzan valores significativos en este índice geoestadístico.

Figura 3. Análisis de punto caliente Gl* de Getis-Ord
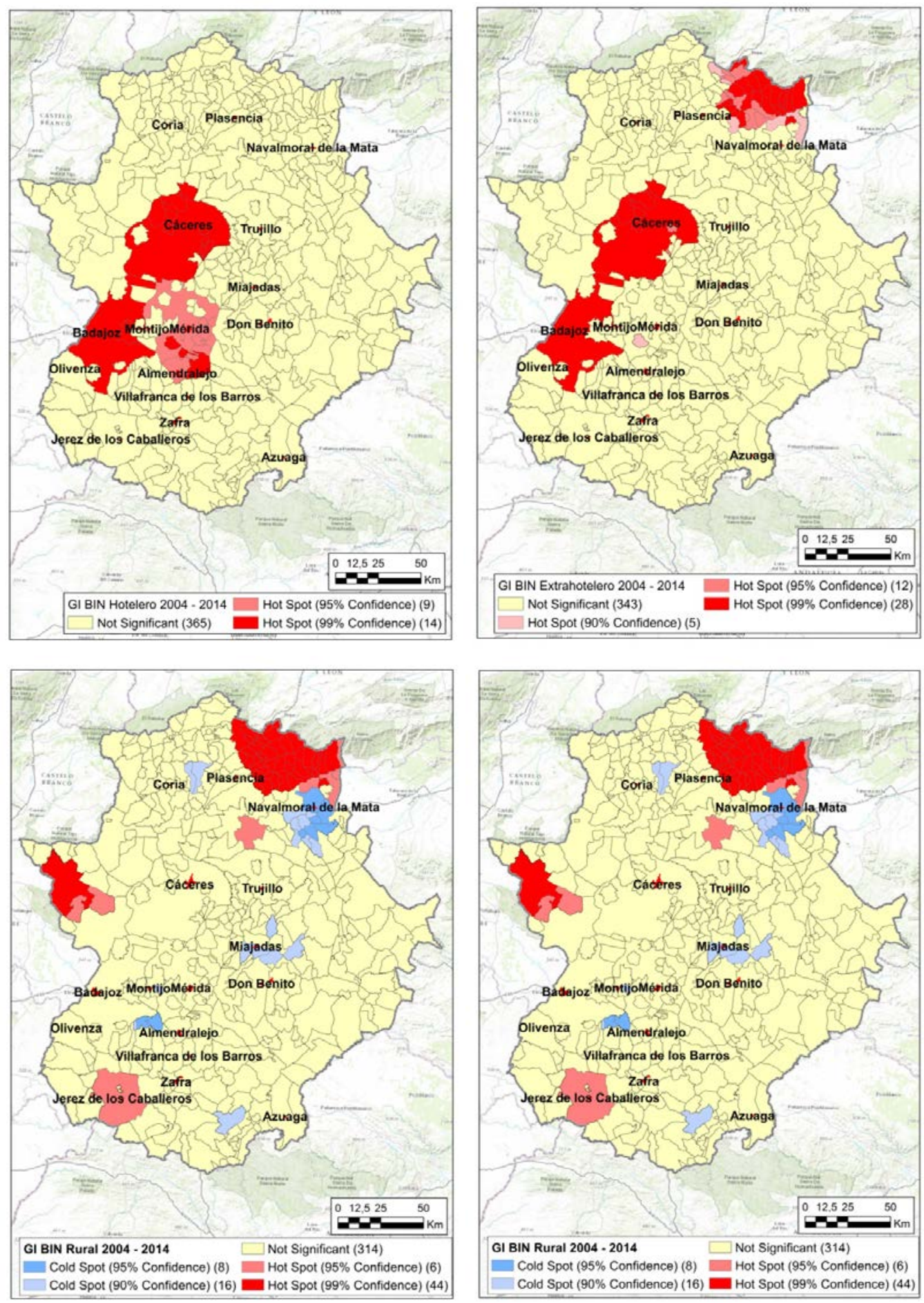

Fuente: elaboración propia

Por su parte, la oferta extrahotelera muestra una situación un poco más compleja, ya que, aparte de los niveles de confianza, se observan dos áreas bien marcadas. Una de ellas, está configurada por 
Badajoz-Cáceres y los municipios de su entorno, sobre todo los de la capital cacereña, en los que se alcanza un nivel de confianza del $99 \%$. La otra, más compleja, abarca los valles del noreste de Extremadura, en los que se forma un interesante punto caliente, claramente definido y con niveles de confianza que tan sólo bajan del 95 \% en la periferia.

El sector del alojamiento rural es, sin ningún género de dudas, el que presenta las relaciones espaciales más complejas. A pesar de que 294 de los 388 municipios no presentan un índice Gi* de agrupación significativa, se detectan puntos calientes y fríos. De entre estos últimos, destaca el desarrollo territorial del conformado por la comarca de lbores, si bien hay otros, de menor entidad espacial, como sucede en Vegas Altas del Guadiana. En ambos casos, los niveles de confianza se sitúan entre el $95 \%$ y el $90 \%$ y afectan a un total de 24 municipios. En contraste, los puntos calientes afectan a 44 municipios, la mayor parte localizados en los valles de La Vera, El Jerte y Ambroz, aunque alcanza en su límite meridional hasta la zona de influencia del parque nacional de Monfragüe. En este vasto punto caliente, el nivel de confianza alcanza el $99 \%$. Existen otros dos puntos calientes, mucho menos significativos desde el punto de vista de la amplitud territorial en la zona de Valencia de Alcántara y su entorno, así como en Jerez de los Caballeros.

Tabla 4. Análisis de punto caliente Gl* de Getis-Ord (resumen de resultados)

\begin{tabular}{|c|c|c|c|c|c|c|c|c|c|}
\hline MUNICIPIO & $\begin{array}{c}\mathrm{GI}^{*} \\
0414 \\
\end{array}$ & $\begin{array}{c}\mathrm{Gl}^{*} \\
\mathrm{HOT}\end{array}$ & $\begin{array}{c}\text { G|* } \\
\text { EXTH }\end{array}$ & $\begin{array}{l}\text { Gl* } \\
\text { RUR }\end{array}$ & MUNICIPIO & $\begin{array}{c}\mathrm{Gl}^{*} \\
0414\end{array}$ & $\begin{array}{c}\mathrm{Gl}^{*} \\
\mathrm{HOT}\end{array}$ & $\begin{array}{c}\text { Gl* } \\
\text { EXTH }\end{array}$ & $\begin{array}{l}\text { Gl* } \\
\text { RUR }\end{array}$ \\
\hline Abadía & 2 & 0 & 1 & 3 & Madrigalejo & 0 & 0 & 0 & -1 \\
\hline Abertura & 0 & 0 & 0 & -1 & Malpartida de Cáceres & 3 & 3 & 3 & 0 \\
\hline Alange & 2 & 3 & 0 & 0 & Mérida & 1 & 2 & 0 & 0 \\
\hline Aldeanueva de la Vera & 1 & 0 & 3 & 3 & Mesas de lbor & 0 & 0 & 0 & -2 \\
\hline Aldeanueva del Camino & 2 & 0 & 2 & 3 & Miajadas & 0 & 0 & 0 & -1 \\
\hline Almaraz & 0 & 0 & 0 & -1 & Millanes & 0 & 0 & 0 & -1 \\
\hline Almendralejo & 0 & 2 & 0 & 0 & Montehermoso & 0 & 0 & 0 & -1 \\
\hline Arroyo de la Luz & 3 & 3 & 3 & 0 & Navaconcejo & 2 & 0 & 3 & 3 \\
\hline Arroyo de San Serván & 3 & 3 & 1 & 0 & Navalmoral de la Mata & 0 & 0 & 0 & -2 \\
\hline Arroyomolinos de la $\mathrm{V}$. & 1 & 0 & 3 & 3 & Pasarón de la Vera & 1 & 0 & 3 & 3 \\
\hline Badajoz & 3 & 3 & 3 & 0 & Peraleda de la Mata & 0 & 0 & 0 & -2 \\
\hline Baños de Montemayor & 3 & 0 & 3 & 3 & Piornal & 1 & 0 & 2 & 3 \\
\hline Barrado & 0 & 0 & 3 & 3 & Pueblonuevo de Miram. & 0 & 0 & 0 & 2 \\
\hline Belvís de Monroy & 0 & 0 & 0 & -1 & Pueblonuevo del Guad. & 2 & 3 & 0 & $\underline{0}$ \\
\hline Bohonal de lbor & 0 & 0 & 0 & -2 & Rebollar & 2 & 0 & 2 & 3 \\
\hline Cabezuela del Valle & 2 & 0 & 3 & 3 & Robledillo de la Vera & 1 & 0 & 3 & 3 \\
\hline Cabrero & 1 & 0 & 3 & 3 & \begin{tabular}{|l} 
San Pedro de Mérida \\
\end{tabular} & 1 & 2 & 0 & 0 \\
\hline Cáceres & 3 & 3 & 3 & 0 & San Vicente de Alcántara & 0 & 0 & 0 & 2 \\
\hline Calamonte & 1 & 2 & 0 & 0 & Santiago del Campo & 3 & 3 & 3 & 0 \\
\hline Campillo de Deleitosa & 0 & 0 & 0 & -1 & Saucedilla & 0 & 0 & 0 & -1 \\
\hline Casar de Cáceres & 3 & 3 & 3 & 0 & \begin{tabular}{|l|} 
Segura de Toro \\
\end{tabular} & 2 & 0 & 1 & 3 \\
\hline Casas del Castañar & 1 & 0 & 3 & 3 & Sierra de Fuentes & 3 & 3 & 2 & 0 \\
\hline Casas del Monte & 2 & 0 & 0 & 3 & Solana de los Barros & 0 & 0 & 0 & -2 \\
\hline Collado de la Vera & 1 & 0 & 2 & 3 & \begin{tabular}{|l|} 
Talavera la Real \\
\end{tabular} & 1 & 3 & 0 & 0 \\
\hline Corte de Peleas & 0 & 0 & 0 & -2 & Talaveruela de la Vera & 0 & 0 & 3 & 3 \\
\hline
\end{tabular}


Tabla 4. Continuación

\begin{tabular}{|c|c|c|c|c|c|c|c|c|c|}
\hline MUNICIPIO & $\begin{array}{c}\mathrm{Gl}^{*} \\
0414\end{array}$ & $\begin{array}{l}\mathrm{Gl}^{*} \\
\mathrm{HOT}\end{array}$ & $\begin{array}{c}\mathrm{Gl}^{*} \\
\text { EXTH }\end{array}$ & $\begin{array}{l}\text { G'* } \\
\text { RUR }\end{array}$ & MUNICIPIO & $\begin{array}{c}\mathrm{G}^{*} \\
0414\end{array}$ & $\begin{array}{l}\text { Gl* } \\
\text { HOT }\end{array}$ & $\begin{array}{c}\mathrm{Gl}^{*} \\
\text { EXTH }\end{array}$ & $\begin{array}{l}\text { Gl* } \\
\text { RUR }\end{array}$ \\
\hline Cuacos de Yuste & 1 & 0 & 2 & 3 & Talayuela & 0 & 0 & 1 & 2 \\
\hline Don Álvaro & 1 & 2 & 0 & 0 & Tejeda de Tiétar & 0 & 0 & 1 & 3 \\
\hline El Torno & 1 & 0 & 2 & 3 & Tiétar & 0 & 0 & 3 & 3 \\
\hline Entrín Bajo & 0 & 0 & 0 & -1 & Tornavacas & 3 & 0 & 3 & 3 \\
\hline Fresnedoso de lbor & 0 & 0 & 0 & -1 & Torrejón el Rubio & 0 & 0 & 0 & 2 \\
\hline Garganta la Olla & 2 & 0 & 3 & 3 & Torremayor & 0 & 0 & 0 & -1 \\
\hline Gargantilla & 2 & 0 & 2 & 3 & Torremejía & 3 & 3 & 0 & 0 \\
\hline Gargüera & 0 & 0 & 0 & 3 & Torremenga & 1 & 0 & 2 & 3 \\
\hline Guijo de Galisteo & 0 & 0 & 0 & -1 & Torreorgaz & 3 & 3 & 0 & 0 \\
\hline Guijo de Santa Bárbara & 3 & 0 & 3 & 3 & Torrequemada & 2 & 3 & 0 & 0 \\
\hline Hervás & 2 & 0 & 2 & 3 & Trujillanos & 1 & 2 & 0 & 0 \\
\hline Higuera & 0 & 0 & 0 & -1 & Valdastillas & 1 & 0 & 2 & 3 \\
\hline Jaraíz de la Vera & 1 & 0 & 2 & 3 & Valdecañas de Tajo & 0 & 0 & 0 & -2 \\
\hline Jarandilla de la Vera & 2 & 0 & 3 & 3 & Valdehúncar & 0 & 0 & 0 & -2 \\
\hline Jerez de los Caballeros & 0 & 0 & 0 & 2 & Valverde de la Vera & 0 & 0 & 3 & 3 \\
\hline Jerte & 3 & 0 & 3 & 3 & Valverde de Mérida & 0 & 2 & 0 & 0 \\
\hline La Garganta & 3 & 0 & 3 & 3 & Viandar de la Vera & 0 & 0 & 3 & 3 \\
\hline La Zarza & 1 & 2 & 0 & 0 & Villagonzalo & 1 & 2 & 0 & 0 \\
\hline Llerena & 0 & 0 & 0 & -1 & Villanueva de la Vera & 0 & 0 & 3 & 3 \\
\hline Losar de la Vera & 1 & 0 & 3 & 3 & Villar de Rena & 0 & 0 & 0 & -1 \\
\hline Madrigal de la Vera & 0 & 0 & 0 & 2 & & & & & \\
\hline
\end{tabular}

Fuente: elaboración propia

\section{Conclusiones}

Tras la realización de este trabajo hay que señalar que destacan las siguientes conclusiones que sirven para corroborar la utilidad que poseen las herramientas geoestadísticas puestas al servicio del análisis turístico. Este conjunto de procedimientos, pese a su complejidad, ofrecen resultados que van más allá del análisis estadístico tradicional, de corte cuantitativo, al considerar el territorio y las relaciones espaciales como un elemento más dentro de los parámetros utilizados y, el turismo se caracteriza precisamente por ese carácter territorial, donde todo está soportado no sólo por el territorio, sino también por la proximidad. En síntesis, del estudio se pueden extraer las siguientes conclusiones:

En primer lugar se observa que la oferta de alojamientos ha aumentado de forma notable desde 2004, incluso durante los años donde la crisis económica ha tenido mayor virulencia, hasta llegar a 2014 con un volumen de plazas de alojamiento notable en los principales destinos turísticos. El aumento ha afectado no sólo a los núcleos que tienen más capacidad para atraer a los turistas, sino también a otros que no disponen de suficientes atractivos para sustentarla, aunque su entorno inmediato sí lo posea. Esta situación motiva que cuando desciende la demanda, como ha sucedido 
durante la crisis económica, los establecimientos turísticos situados en algunos espacios tengan dificultades para mantener niveles de rentabilidad aceptables.

En segundo lugar se observa la complejidad que tiene la geoestadística, mucho mayor que la ya de por sí compleja relación que se establece entre variables, pues el condicionante territorial impone una forma específica de relación. En este sentido, el abanico de posibilidades es enorme, pues el carácter de vecindad, su relación en definitiva, dependerá de la propia localización de los núcleos de población sobre los que se asienta la oferta, con las trabas que ello conlleva. Éstas son mayores si cabe en zonas como Extremadura, donde coexisten poblaciones muy próximas y muy alejadas, aunque se utilice la distancia euclidiana. Afortunadamente, el software utilizado ha permitido calcular distancias idóneas teniendo en cuenta el factor de localización y los parámetros analizados.

En tercer lugar cabe mencionar que, de todas las formas de relación posible, tras numerosos ensayos y dada la casuística del sistema analizado, se estima que el criterio de distancia es el más adecuado para determinar las relaciones que se establecen entre los diferentes núcleos, ya que se han utilizado este tipo de entidades territoriales como las más rigurosas, pese a que el grado de explicación obtenido sea menor. Obviamente, para que la representación se aprecie mejor, se ha optado por representar estos valores en cada término municipal, no en el núcleo. Esta decisión se entiende si se tiene en cuenta que al utilizar los términos municipales, se incrementa de forma exponencial el criterio de relación espacial, debido a que algunos, como Cáceres, Badajoz o Mérida, ocupan 1768, 1470 ○ $865 \mathrm{~km}^{2}$ respectivamente, con lo que los "vecinos" estarían muy distantes.

Por último, se destaca que utilizando cualquiera de los dos parámetros, ya sea para descubrir agrupaciones o valores atípicos, ya sea para detectar la presencia de puntos calientes o fríos, los resultados que se obtienen son muy parecidos y corroboran en gran medida el contrapuesto desarrollo turístico que tiene Extremadura. 


\section{Bibliografía}

Anselin, L. (1995). Local Indicators of Spatial Association (LISA). Geographical Analysis, 27(2), 93-115.

Antón, S., y González, F. (2011). Planificación territorial del turismo. Barcelona: UOC.

Archer, B., Cooper, C., y Ruhanen, L. (2012). The positive and negative impacts of tourism. En W. Theobald, Global Tourism (pp. 79-102). Nueva York: Routledge.

Baloblu, S., y McCelary, K. (1999). A model of destination image formation. Annals of tourism research, 26(4), 868-897.

Buckley, R. (2011). Tourism and Environment. Annual Review of Environment and Resources, 36, 397-416.

Cawley, M., y Gillmor, D. (2008). Integrated rural tourism: Concepts and Practice. Annals of Tourism Research, 35(2), 316-337.

Consejería de Fomento, Vivienda y Ordenación del Territorio (2014). Anuario 2014. Mérida: G. d. Extremadura.

Díaz Viera, M. (2002). Geoestadística aplicada. México: UNAM.

Dredge, D., y Jamal, T. (2015). Progress in tourism planning and policy: A post-structural perspective on knowledge production. Tourism Management, 51, 285-297.

Fang, Y., Huang, Z., Wang, K., y Cai, B. (2015). Spatial Pattern of Chinese Tourism Development and Its Mechanism Based on Different Spatial-temporal Scales: Taking the Panel Data of China Mainland (1996-2010) for Example. Journal of Landscape Research, 7(2), 47-54.

García-Palomares, J., Gutiérrez, J., y Mínguez, C. (2015). Identification of tourist hot spots based on social networks: A comparative analysis of European metropolises using photo-sharing services and GIS. Applied Geography, 63, 408-417.

Getis, A., y Ord, J. (1992). The Analysis of Spatial Association by Use of Distance Statistics. Geographical Analysis, 24(3), 189-206.

Grinberger, A., Shoval, N., y McKercher, B. (2014). Typologies of tourists' time-space consumption: a new approach using GPS data and GIS tools. Tourism Geographies, 16(1), 105 123.

Gunn, C. (1988). Tourism planning. Lond: Taylor \& Francis.

Haggett, P. (1976). Análisis locacional en la geografía humana. Barcelona: Gustavo Gili. 
Hall, C. (2008). Tourism planning: policies, processes and relationships. Harlow: Pearson/Prentice Hall.

Hall, C. (2011). Policy learning and policy failure in sustainable tourism governance: from first- and second-order to third-order change? Journal of Sustainable Tourism, 19(4-5), 649-671.

Hall, C., y Page, S. (2009). Progress in Tourism Management: From the geography of tourism to geographies of tourism. A review. Tourism Management, 30(1), 3-16.

Hall, M., y Page, S. (2014). The geography of tourism and recreation: Environment, place and space. Nueva York: Routledge.

INE (2015). Contabilidad Regional de España. Extremadura. Recuperado el 25 de septiembre de 2015, de http://www.ine.es/daco/daco42/cre00/b2008/dacocre_base2008.htm

Inskeep, E. (1991). Tourism planning: an integrated and sustainable development approach. New York: Van Nostrand Reinhold.

Jordan, E. (2015). Planning as a Coping Response to Proposed Tourism Development. Journal of Travel Research, 54(3), 316-328.

Lattin, J., Carrol, J., y Gren, P. (2003). Analyzing multivariate data. Pacific Grove, CA: Thomson/Brooks/Cole.

Lee, S. H., Choi, J. Y., Yoo, S. H., y Oh, Y. G. (2013). Evaluating spatial centrality for integrated tourism management in rural areas using GIS and network analysis. Tourism Management, 34, 1424.

Mason, P. (2015). Tourism Impacts, Planning and Management. New York: Routledge.

Mathieson, A., y Wall, G. (1982). Tourism, economic, physical and social impacts. Harlow: Longman.

Mohammed, I., Guillet, B., y Law, R. (2015). The contributions of economics to hospitality literature: A content analysis of hospitality and tourism journals. International Journal of Hospitality Management, 44, 99-110.

Morpeth, N., y Yan, H. (2015). Approaches to planning and tourism. En H. Yan, y N. Morpeth (Eds.), Planning for tourism: towards a sustainable future (pp. 3-23). Wallingford: CABI.

Nieto, A. y Gurría, J. L. (2008). Las políiticas rurales europeas y su impacto en Extremadura. Boletín de la Asociación de Geógrafos Españoles, 48, 225-246.

Ofobruku, A., Onabanjo, N., y Iheabunike, B. (2013). Utilisation of GIS technology for tourism management in Victoria Island Lagos. European Scientific Journal, 9(3), 92-118. 
Olivera, A. (2011). Patrimonio inmaterial, recurso turístico y espíritu de los territorios. Cuadernos de Turismo, 27, 663-677.

Polo, A. I. y Chica, J. (2015). Market Orientation Adoption among Rural Tourism Enterprises: The Effect of the Location and Characteristics of the Firm. International Journal of Tourism Research, 17(1), 54-65.

Polo, A. I., Frías, M. D., Rodríguez, M. A. y Chica, J. (2015). Rural lodging establishments: effects of location anda internal resources and characteristics on room rates. Tourism Geographies, 17(1), 91-111.

Pulido, M., Lagar, D., y García, R. (2011). El geoturismo como estrategia de desarrollo en áreas deprimidas: Propuesta del Geoparque Villuercas, Ibores, Jara (Extremadura). Boletín de la Asociación de Geógrafos Españones, 56, 485-498.

Rengifo, J. I., Campesino, A. J., y Sánchez, J. M. (2015). El turismo en la ciudad de Cáceres (1986-2010): un cuarto de siglo emblemático. Boletín de la Asociación de Geógrafos Españoles, $67(1), 907-928$.

Quintero Rivera, A. (2002). Salsa, identidad y globalización. Redefiniciones caribeñas a las geografías y al tiempo. Recuperado el 18 de 9 de 2015 de http://www.redalyc.org/pdf/822/82200609.pdf

Ritchie, B., Burns, P., y Palmer, C. (2005). Tourism Research Methods: Integrating Theory with Practice. Wallingford: CABI.

Rutherford, J., Kobryn, H., y Newsome, D. (2015). A case study in the evaluation of geotourism potential through geographic information systems: application in a geology-rich island tourism hotspot. Current Issues in Tourism, 18(3), 267-285.

Rutty, M., Gössling, S., Scott, D., y Hall, C. (2015). The global effects and impacts of tourism. En C. Hall, y S. Gossling, The Routledge Handbook of Tourism and Sustainability (pp. 36-62). Nueva York: Routledge.

Sánchez Martín, J. (2013). La evaluación del potencial para el desarrollo del turismo rural. Aplicación metodológica sobre la provincia de Cáceres. GeoFocus, 13(1), 99-130.

Sánchez, J. M.; Gurría, J. L.; Leco, F., y Pérez, Mª N. (2001). SIG para el desarrollo turístico en los espacios rurales de Extremadura. Estudios Geográficos, 243, 335-368.

Sánchez, J.M., Pérez, Ma. N., Jurado, J. C., y Granados, Mª. M. (1999) Detección de áreas óptimas para la implantación de alojamientos rurales. Una aplicación SIG. Lurralde. Investigación y Espacio, 22, 367-384. 
Sánchez, J. M., Sánchez, M., y Rengifo, J. I. (2013). La evaluación del potencial para el desarrollo del turismo rural. Aplicación metodológica sobre la provincia de Cáceres. GeoFocus, 13(1), 99-130.

Sánchez Rivero, M. (2008). Análisis espacial de datos y turismo: nuevas técnicas para el análisis turístico. Una aplicación al caso extremeño. Revista de Estudios Empresariales. Segunda época, 2, 48-66.

Santana Turégano, M. Á. (2003). Formas de desarrollo turístico, redes y sutuación de empleo. El caso de Maspalomas (Gran Canaria) (Tesis doctoral). Barcelona. Recuperado de http://www.tdx.cat/bitstream/handle/10803/5116/mast1de2.pdf

Sarrión, M. D., Benítez, M.D., y Mora, E. O. (2015). Spatial distribution of tourism supply in Andalusia. Tourism Management Perspectives, 15, 29-45.

Simmons, D. (1994). Community participation in tourism planning. Tourism Management, 15(2), 98-108.

Smith, V. (2012). Hosts and Guests: The Anthropology of Tourism. Phyladelphia: University of Pennsylvania Press.

Song, H., Dwyer, L., y Zheng, G. (2012). Tourism economics research: A review and assessment. Annals of Tourism Reseach, 36(3), 1653-1682.

Stylidis, D., Srit, J., y Biran, A. (2014). An Exploratory Study of Residents' Perception of Place Image. Journal of Travel Research, 10, 1-16.

Tobler, W. (1970). A Computer Movie Simulating Urban Growth in the Detroit Region. Economic Geography, 46, 234-240.

Yokeno, N. (1968). La localisation de l'industrie touristique: application de l'analyse de ThunenWeber. Cahiers de tourisme, 9, 18.

Zhou, Y., Maumbe, K., Deng, J., y Selin, S. (2015). Resource-based destination competitiveness evaluation using a hybrid analytic hierarchy process (AHP): The case study of West Virginia. Tourism Management Perspectives, 15, 70-80. 